\title{
Cholesterol-conjugated let-7a mimics: antitumor efficacy on hepatocellular carcinoma in vitro and in a preclinical orthotopic xenograft model of systemic therapy
}

Yang Ming Liu' ${ }^{1}$, Yu Xia ${ }^{2}$, Wei Dai ${ }^{3}$, Hua Ye Han ${ }^{3}$, Yu Xue Dong ${ }^{4}$, Jiong Cai ${ }^{5}$, Xuan Zeng ${ }^{1}$, Feng Yu Luo ${ }^{1}$, Tao Yang ${ }^{1}$, Yuan Zhi Li', Jie Chen ${ }^{1 *}$ and Jian Guan ${ }^{1,6^{*}}$

\begin{abstract}
Background: A major challenge to the clinical utility of let-7 for hepatocellular carcinoma (HCC) therapy is the lack of an effective carrier to target tumours. We confirmed the high transfection efficiency of cholesterol-conjugated let-7a miRNA mimics (Chol-let-7a) in human HCC cells, as well as their high affinity for liver tissue in nude mice. However, their antitumor efficacy via systemic delivery remains unknown.

Methods: We explored the effects of Chol-let-7a on HCC in vitro and in vivo. Cell viability and mobility, let-7a abundance and the target ras genes was measured. Live-cell image and cell ultrastructure was observed. Antitumor efficacy in vivo was analyzed by ultrasonography, hispatholgogy and transmission electronic microscopy in a preclinical model of HCC orthotopic xenografts with systemic therapy.

Results: Chol-let-7a inhibited the viability and mobility of HCC cells. Chol-let-7a was primarily observed in the cytoplasm and induced organelle changes, including autophagy. Mild changes were observed in the cells treated with negative control miRNA. Chol-let-7a reached HCC orthotopic tumours, significantly inhibited tumour growth, and prevented local invasion and metastasis. Compared to control tumours, Chol-let-7a-treated tumours showed more necrosis. Tumour cells showed no significant atypia, and mitoses were very rare after systemic Chol-let-7a therapy. Furthermore, let-7a abundance in orthotopic xenografts was coincident with a reduction in the expression of 3 human ras mRNAs and RAS proteins.
\end{abstract}

Conclusions: Chol-let-7a exerted significant antitumor effects by down-regulating all human ras genes at the transcriptional and translational levels. Chol-let-7a inhibited cell proliferation, growth, and metastasis, and mainly functioned in the cytoplasm. Chol-let-7a represents a potential useful modified molecule for systemic HCC therapy.

\section{Background}

Hepatocellular carcinoma (HCC) is the fifth most common cancer worldwide and the third most common cause of cancer mortality and has high recurrence rates after surgery. Chemotherapy and radiotherapy for HCC show limited efficacy and serious toxicity [1,2]. New therapeutic strategies are urgently needed, particularly for the treatment of advanced tumours.

\footnotetext{
* Correspondence: xhblk@163.com; gjpumch@126.com

'Department of Pathology, Peking Union Medical College (PUMC) Hospital, PUMC \& Chinese Academy of Medical Sciences (CAMS), Beijing, China

${ }^{6}$ Department of Scientific Research, Peking Union Medical College (PUMC) Hospital, PUMC \& Chinese Academy of Medical Sciences (CAMS), Beijing, China Full list of author information is available at the end of the article
}

MicroRNAs (miRNAs) are endogenous non-coding small RNAs that repress gene expression at the posttranscriptional level by base pairing to the 3 '-untranslated region of target messenger RNAs, and they have been identified as important mediators of carcinogenesis and clinical prognosis [3-6]. The most recent findings regarding the role of miRNAs in HCC confirmed that they hold promise as new tools for diagnosis and therapy [7-11]. A recent study in C. elegans reports that the let-7 family negatively regulates let-60/RAS, and also that the let-60/RAS 3'-UTRs, including the 3'-UTRs of the human ras genes, contain multiple let-7 complementary sites (LCSs), which allow let-7 to regulate RAS protein 
expression [12]. Furthermore, let-7 has been reported to inhibit tumour growth by down-regulating KRAS in some cancers, such as pancreatic carcinoma and lung cancer [13,14]. Analysis with a computational screen showed that the human $n$-ras, $k$-ras, and $h$-ras mRNA 3 '-UTRs have 9,8 , and 3 potential LCSs, respectively [12]. Although ras proto-oncogenes produced by mutations in codons 12,13, and 61 do not play major roles in hepatocellular carcinogenesis [15], abnormal activation of the RAS pathway occurs in human HCC, and activated (GTP-bound) Pan-RAS, HRAS, KRAS, and NRAS are significantly up-regulated in human hepatocarcinogenesis $[16,17]$. Thus, we hypothesize that modulation of let-7 expression and its target RAS is a promising strategy for HCC treatment, because let-7 might suppress HCC tumour growth by down-regulating all human ras genes.

Recently, antitumor effects of synthetic miRNA mimics were confirmed in vitro and in vivo [18-20]. Hou et al. showed that intratumoural administration of cholesterolconjugated PAK4 siRNA suppressed subcutaneous tumour growth in the SMMC-LTNM model [21]. Trang and colleagues [18] found that synthetic miR-34a and let-7 mimics caused lung tumour reduction in mice. However, these mimics did not produced high miRNA levels in the liver tissues. We confirmed the significantly higher transfection efficiency of cholesterol-conjugated let-7a miRNA mimics (Chol-let-7a) in human HCC cells in vitro. Given the observed high affinity of Chol-let-7a for liver tissue in nude mice, we hypothesize that Chol-let-7a may be an ideal modified molecule for systemic HCC therapy.

In this study, we explored the effects of Chol-let-7a on HCC tumour cells in vitro, as well as its antitumor efficacy in an in vivo preclinical model of HCC orthotopic xenografts, to evaluate its potential as a systemically administered drug in the treatment of HCC. In addition, we explored the effects of Chol-let-7a on ras gene expression at the transcriptional and translational levels.

\section{Methods}

Materials and methods

Cell culture and mice

HepG2 and SMMC7721 cells were cultured in DMEM (Invitrogen, Carlsbad, CA, USA) supplemented with $10 \%$ foetal bovine serum (Invitrogen) and pen/strep $(100 \mu \mathrm{g} / \mathrm{mL})$. BALB/c nude $(\mathrm{nu} / \mathrm{nu})$ mice (6-7 weeks old, $20 \pm 3 \mathrm{~g}$ ) were purchased from the National Institutes for Food and Drug Control (lot number: 11400500001092; Beijing, China).

\section{MTT cell proliferation assays}

Cholesterol-conjugated let-7a mimics (Chol-let-7a) and the negative control miRNA (Chol-miRCtrl) were purchased from Ribobio (Guangzhou, China). Cells $\left(5 \times 10^{3}\right)$ were cultured in 96-well flat-bottomed plates. After $24 \mathrm{~h}$ of cell culture, cells were transfected with $50 \mathrm{nM}$ Chol-let-7a or Chol-miRCtrl according to manufacturer instructions. Cells were cultured in $100 \mu \mathrm{L}$ DMEM containing 10\% FBS and $20 \mu \mathrm{L}$ MTS reagent powder (Promega, Madison, WI, USA). Cells were harvested and seeded on 96-well flat-bottomed plates, which were incubated at $37^{\circ} \mathrm{C}$ for $4 \mathrm{~h}$. After incubation for 1, 2, 3, 4, or 5 days, the absorbance at $550 \mathrm{nM}$ was determined for each well.

\section{Invasion and migration assay}

Assays of invasion and migration were performed as described in previous report [22]. For invasion assays, $5 \times 10^{4}$ cells in serum-free media were seeded into the upper chambers of a 24-well BioCoat Matrigel invasion chamber (Becton Dickinson Labware, Franklin Lakes, NJ, USA) with an $8-\mu \mathrm{m}$ pore polycarbonate membrane coated with Matrigel. For migration assays, $5 \times 10^{4}$ cells were seeded into the upper chambers of a 24-well BioCoat control insert (Becton Dickinson Labware, Franklin Lakes, NJ, USA) with uncoated $8-\mu$ m pores in serum-free media. Medium with $10 \%$ FBS was added to the lower chambers as a chemoattractant. After $24 \mathrm{~h}$ of incubation, cells remaining on the upper surface of the membrane were removed with a cotton swab and cells that invaded through the membrane filter were fixed with $100 \%$ methanol, stained by hematoxylin and eosin, and photographed by soft BioLife DP under a microscope (Olympus BX40 with a DP70 digital camera, Tokyo, Japan). The number of invading or migrating cells was manually counted per high-power field for each condition (eight fields on each membrane were randomly selected).

\section{Wounding assay}

Cells were grown to confluence in $25 \mathrm{~cm}^{2}$ cell culture flasks. Artificial wound tracks were created by scraping confluent cell monolayers with a pipette tip. After removal of the detached cells by gentle washing with PBS, the cells were fed with fresh complete medium and incubated to allow cells to migrate into the open area. The ability of the cells to migrate into the wound area was assessed at 24,48 , and $72 \mathrm{~h}$ after scratching by comparing the wound tracks in micrographs of 3 randomly selected wound areas.

\section{Quantitative real-time PCR and reverse transcription PCR}

Total miRNA from HCC cells or snap-frozen HepG2 xenografts was isolated using the mirVANA ${ }^{\mathrm{Tm}}$ PARIS $^{\mathrm{Tm}}$ RNA isolation kit (Applied Biosystems, Carlsbad, CA, USA). RNA (10 ng) was reverse-transcribed with the miRNA Reverse Transcription Kit (Applied Biosystems) and let-7a specific primers (TaqMan miRNA assay, Applied Biosystems).

Total RNA was extracted from HCC cells or snapfrozen HepG2 xenografts using the IllustraRNA spin 
Mini RNA Isolation Kit (GE Healthcare UK Limited, Amersham Place, Little Chalfont, UK). cDNA was synthesized using SuperScript TM III First-Strand Synthesis SuperMix for quantitative real-time reverse transcription PCR (qRT-PCR; Invitrogen Corporation, Carlsbad, CA, USA) and primers specific for the 3 human ras genes (TaqMan miRNA assay, Applied Biosystems).

Quantitative PCR was performed using RNU6 or GAPDH as a housekeeping control with an ABI Prism 7500 Sequence Detection System (Perkin-Elmer Applied Biosystems, Foster City, USA) and the Perkin-Elmer Biosystems analysis software in a manner consistent with the manufacturer's instructions. Relative expression was calculated using the $2^{-\Delta \Delta C \mathrm{~T}}$ method [23].

\section{Western blotting}

HCC cells and tissues from snap-frozen HepG2 xenografts were lysed using RIPA lysis buffer (Applygen Technologies, Beijing, China). Proteins were quantified using a BCA protein kit (Applygen). Proteins $(50 \mu \mathrm{g})$ were separated by SDS-PAGE and transferred to an Immobilon-P membrane (Millipore, Billerica, MA, USA). The membranes were blocked in 5\% non-fat milk and incubated with primary antibodies. The membranes were washed in PBS-T (PBS and $0.1 \%$ Tween-20) and incubated with a peroxidaseconjugated secondary antibody (KPL, Gaithersburg, MD, USA), followed by development with a chemiluminescent substrate (Applygen). The Gel-Doc imaging system was used to scan images on Kodak film. Antibodies for KRAS, HRAS, and NRAS were purchased from Santa Cruz Biotechnology (Santa Cruz, CA, USA). GAPDH and beta-actin ( $\beta$-action) antibodies were obtained from Proteintech (Chicago, IL, USA).

\section{Transfection, live-cell imaging, and transmission electron microscopy}

HepG2 and SMMC771 cells were labelled with GFP. Chol-let-7a and negative control mimics labelled with Cy5 were purchased from Ribobio (Guangzhou, China).

The GFP-labelled cells $\left(2-3 \times 10^{4}\right)$ were seeded in 8-well BD Falcon ${ }^{\text {TM }}$ and BD BioCoat ${ }^{\text {TM }}$ Culture Slides (Becton Dickinson Labware, Franklin Lakes, NJ, USA). After 48 h, cells were transfected with Cy5-labelled Chol-let-7a or the negative control mimics (Chol-miRCtrl).

For live-cell imaging, cells were continuously observed using a PerkinElmer UltraVIEW VoX-3D Live Cell Imaging System (Shanghai, China) from 24 to $72 \mathrm{~h}$ post-transfection. Digital images were produced using Volocity Demo software (version 5.4, 32-bit). Colocalization events were calculated using the Volocity Demo software as described in the manufacturer's recommendations. The experiment was repeated 3 times and all samples for each individual experiment were scanned at 5 different locations.
For electron microscopy, cells were collected at $48 \mathrm{~h}$ and $60 \mathrm{~h}$ after transfection and were fixed with $2.5 \%$ glutaraldehyde for $30 \mathrm{~min}$ at room temperature, followed by $1.5 \mathrm{~h}$ in $2 \% \mathrm{OsO}_{4}$. Samples were stained and examined with a transmission electron microscope (JEOL JEM 1010, Tokyo, Japan), and digital images were obtained with an Erlangshen ES1000W camera (Model 785, Gatan, Warrendale, PA, USA).

\section{In vivo experiments}

All procedures were performed in accordance with the Guide for the Care and Use of Laboratory Animals (NIH publication nos. 80-23, revised 1996) and with the experimental animal welfare ethics regulations of China, with the approval of the Institution Animal Care and Use Committee of Peking Union Medical College Hospital. All animal experiments were performed at the Centre for Experimental Animal Research (CEAR), Institute of Basic Medical Sciences (IBMS), CAMS \& PUMC.

\section{Orthotopic xenograft model with nude mice and systemic therapy with Chol-let-7a}

HepG2 cells $\left(2 \times 10^{6}\right)$ were injected directly into the livers of 20 nude mice. One week later, 18 mice with successfully engrafted HepG2 orthotopic xenografts were randomized into 3 groups of 6 animals each and examined by ultrasonography in a double-blinded manner (VisualSonics, Inc., Toronto, Ontario, Canada).

Two cohorts were treated with $5 \mathrm{nmol}$ of Chol-let-7a or the negative control mimic (Chol-miRCtrl) in $250 \mu \mathrm{L}$ saline buffer (Ribobio, Guangzhou, China) as suggested by the instruction manual. Another cohort was treated with saline buffer alone (blank). Systemic therapy was administered via the tail vein every 3 days for 6 weeks. Orthotopic tumour size in the liver and potential secondary metastases in the spleen were confirmed by ultrasonography with a Vevo 2100 high-frequency ultrasound system (VisualSonics, Inc., Toronto, Ontario, Canada) with measurements in 3 orthogonal axes $(a, b$, and $c$ ). Tumour volumes were determined as $V=(a b c) / 2$ [24]. The presence of tumours was confirmed via 2dimensional vertical interfaces. Whole-animal imaging was recorded using a Kodak FX Pro in vivo imaging system. The xenograft growth curves of the 3 groups were based on the mean volume of 6 samples weekly, and inhibition was calculated based on the volume 5 weeks (the tumours of the 2 control groups at week 6 were too large for ultrasonography) after treatment as follows:

Inhibitory rate $(\%)=1-$ mean volume of the treatment group/mean volume $($ blank $) \times 100$

At the culmination of therapy, tumour tissues were harvested and preserved in 10\% neutral buffered 
formalin for pathology observation. Fresh tumour tissues were snap-frozen for qRT-PCR, western blotting, or transmission electron microscopy.

\section{Statistical analysis}

Data are expressed as the mean \pm SEM. All data analyses were performed with SPSS 16.0 software (IBM, Inc., Armonk, NY, USA). Analysis of variance (ANOVA) and Student's t-test were used for statistical comparisons between groups. $p<0.05$ was considered to be statistically significant.

\section{Results}

\section{Chol-let-7a reduced HCC cell growth and viability in vitro}

The growth curves of HepG2 and SMMC7721 cells during the MTT assay are shown in Figure $1 \mathrm{~A}$ and B, respectively. After 5 days, Chol-let-7a decreased HepG2 and SMMC7721 viability by $37.7 \%$ and $36.6 \%$, respectively, in comparison with the parental cells (blank) $(p<0.05)$. No significant differences in growth were observed among the 2 control HCC cell lines, the negative control miRNA mimic (Chol-miRCtrl)-treated cells, and the parental cells $(p>0.05)$. These results verified that Chol-let-7a inhibited $\mathrm{HCC}$ cell growth in vitro.

\section{Chol-let-7a inhibited the migration and invasion of HCC cells in vitro}

We determined the effects of Chol-let-7a on HCC cell migration and invasion, which are 2 key steps in tumour metastasis. HepG2 and SMMC7721 cells were transfected with Chol-let-7a or with Chol-miRCtrl as a negative miRNA control. The transfected Chol-let-7a and Chol-miRCtrl cells and the parental cells were used in the migration and invasion assay $48 \mathrm{~h}$ post-transfection.

A chamber-based cell migration assay revealed that the number of Chol-let-7a-treated HCC cells that migrated through the membrane was significantly lower than the number of Chol-miRCtrl-treated cells $(p<0.05)$ or parental cells (blank) $(p<0.05)$ that migrated through the membrane (HepG2, $148.3 \pm 7.02$ (Chol-let-7a) vs. $203.0 \pm 5.29$ (Chol-miRCtrl) vs. $214.67 \pm 11.67$ (blank); SMMC7721, $155.67 \pm 6.66$ (Chol-let-7a) vs. $218.33 \pm 9.45$ (Chol-miRCtrl) vs. $230.67 \pm 7.02$ (blank)) (Figure 1C). There were no significant differences between the 2 control groups $(p>0.05)$, indicating that Chol-let-7a suppressed HCC cell migration.

We also used an in vitro wound healing assay to measure cell migration (data not shown). Healing speed was slower and gaps were wider in the Chol-let-7a-treated HepG2 and SMMC7721 cells at each time point (24, $48 \mathrm{~h}$, and $72 \mathrm{~h}$ ) in comparison with their respective control groups. At $48 \mathrm{~h}$, most gaps in the 2 control cell groups were completely closed; whereas the gaps in the Chol-let-7a-treated cells remained open. Consistent with the results from the chamber-based cell migration assay, these data indicated that Chol-let-7a inhibited HCC cell migration.

Next, we evaluated the ability of HCC cells to pass through the extracellular matrix (ECM) in a Boyden chamber invasion assay. We found significantly fewer invading cells in the Chol-let-7a-treated group in comparison with the 2 control groups (Figure 1D) $(66.33 \pm 4.73$ (Chol-let-7a) vs. $91.33 \pm 3.21$ (Chol-miRCtrl) vs. $97.00 \pm 5.29$ (blank); Chol-let-7a vs. the 2 control groups, both $p<0.05)$, and similar results were observed with SMMC7721 cells (73.00 \pm 5.29 (Chol-let-7a) vs. $91.33 \pm 3.21$ (Chol-miRCtrl) vs. $103.33 \pm 4.73$ (blank); Chol-let-7a vs. the 2 control groups, both $p<0.05)$. Thus, it appears that Chol-let-7a affects cell migration and invasion.

\section{Up-regulated let-7a down-regulated ras/RAS expression in HCC cells}

We measured let-7a levels by quantitative real-time PCR $48 \mathrm{~h}$ after transfection with Chol-let-7a and Chol-miRCtrl. Using miRNA-specific primers, let-7a up-regulation in comparison with parental HCC cells and Chol-miRCtrltreated control cells was confirmed in Chol-let-7a-treated cells (see Additional file 1A). Next, we analysed the expression of let-7 target ras genes at the transcriptional and translational levels $48 \mathrm{~h}$ after transfection. Western blotting revealed a marked decrease in KRAS, HRAS, and NRAS protein abundance in the Chol-let-7a-treated HepG2 and SMMC7721 cells (see Additional file 1B). Quantitative real-time PCR (qRT-PCR) was used to measure $k$-ras, $h$-ras, and $n$-ras transcript abundance in the Chol-let-7a-treated HCC cells, and these 3 ras genes were found to be reduced by Chol-let-7a treatment (see Additional file $1 C$ ). These results verified our hypothesis that Chol-let-7a would inhibit the transcription and translation of all 3 human ras genes in vitro.

\section{Live-cell images}

Images of live HCC cells were taken after treatment with Chol-let-7a or the negative control miRNA (Chol-miRCtrl). The parental cells served as blank controls that were visually inspected to evaluate potential off-target interactions of Chol-miRCtrl. Living HepG2 and SMMC7721 cells labelled by GFP were identified by green fluorescence. Images taken at the various observation time points are shown in Figure 2. The red fluorescence that indicated Chol-let-7a and Chol-miRCtrl was primarily focused in the cytoplasm (Figure 2). Through analysis of live images, we found that most of the Chol-let-7a-treated cells lost GFP fluorescence earlier than the 2 control groups (Figure 2). Some Chol-let-7a-treated cells showed typical features of apoptosis (Figure 2, Additional file 2). The numbers of GFP positive cells in the Chol-let-7a, Chol-miRCtrl, and 


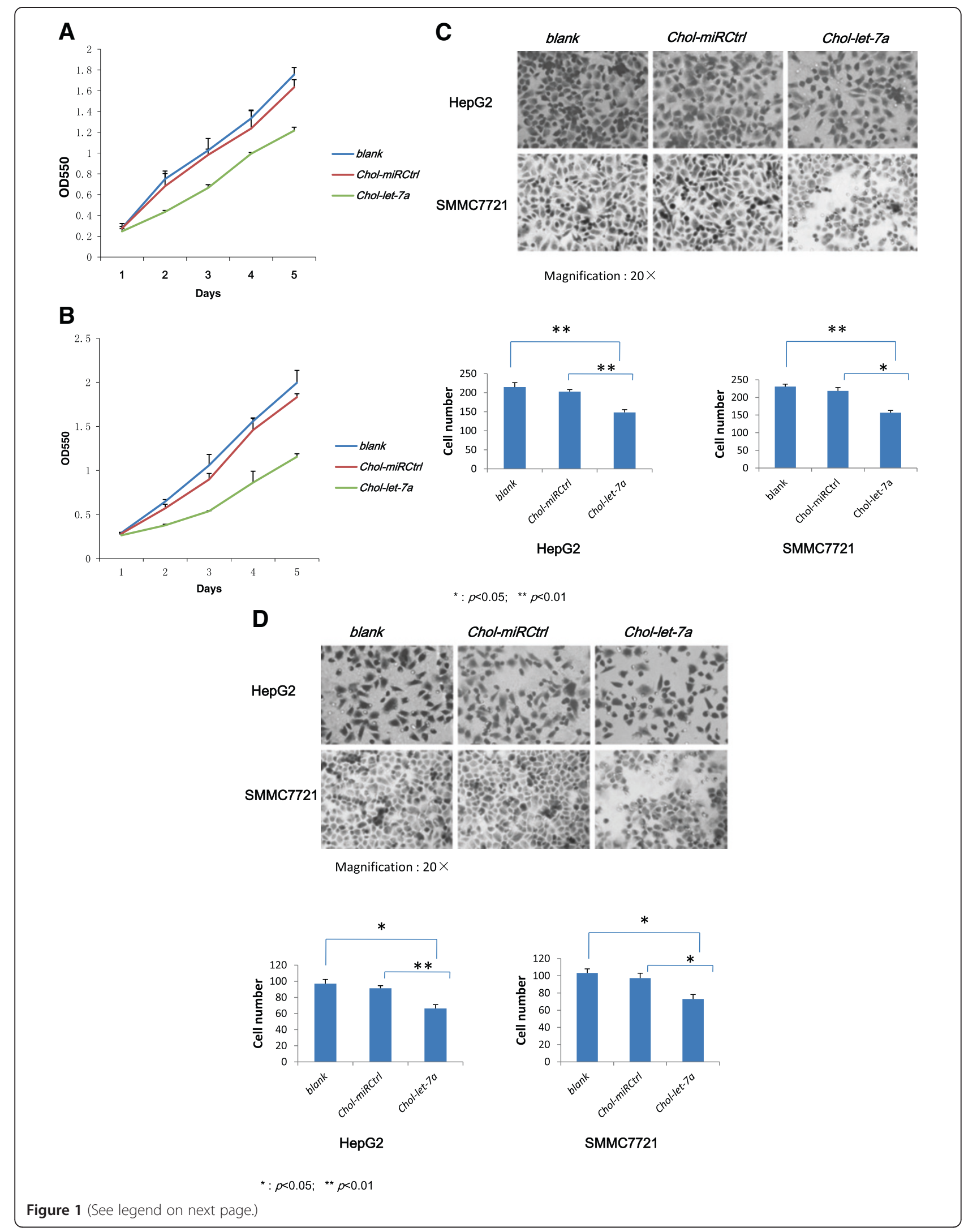




\begin{abstract}
(See figure on previous page.)
Figure 1 Chol-let-7a inhibited HCC cell growth and cell viability in vitro. A,B: MTT assays show the effects of Chol-let-7a and Chol-miRCtrl on the growth of HepG2 and SMMC7721 cells. The figure shows the growth curve of tumour cells from days 1-5. blank, parental cells; Chol-miRCtrl, Chol-miRCtrl-transfected cells; Chol-let-7a, Chol-let-7a-transfected cells. C: A chamber-based cell migration assay showing that Chol-let-7a inhibits the migration of HCC cells. HepG2: Chol-let-7a vs. Chol-miRCtrl vs. blank, $148.3 \pm 7.02$ vs. $203.0 \pm 5.29$ vs. $214.67 \pm 11.67$; T-test: Chol-let-7a vs. Chol-miRCtrl, $p=0.001$; Chol-let-7a vs. blank, $p=0.005$; Chol-miRCtrl vs. blank, $p=0.207$. SMMC7721: Chol-let-7a vs. Chol-miRCtrl vs. blank, $155.67 \pm$ 6.66 vs. $218.33 \pm 9.45$ vs. $230.67 \pm 7.02$; T-test: Chol-let-7a vs. Chol-miRCtrl, $p=0.021$; Chol-let-7a vs. blank, $p=0.01$; Chol-miRCtrl vs. blank, $p=0.066$. Magnification: 20× D: The inhibitory effect of Chol-let-7a on cell invasion ability in the Boyden chamber invasion assay. HepG2: Chol-let-7a vs. Chol-miRCtrl vs. blank, $66.33 \pm 4.73 v s .91 .33 \pm 3.21$ vs. $97.00 \pm 5.29$; T-test: Chol-let-7a vs. Chol-miRCtrl, $p=0.005$; Chol-let-7a vs. blank, $p=0.026$; Chol-miRCtrl vs. blank, $p=0.362$. SMMC7721 cells: Chol-let-7a vs. Chol-miRCtrl vs. blank: $73.00 \pm 5.29$ vs. $91.33 \pm 3.21$ vs. 103.33 \pm 4.73 . T-test: Chol-let-7a vs. Chol-miRCtrl, $p=0.019$; Chol-let-7a vs. blank, $p=0.016$; Chol-miRCtrl vs. blank, $p=0.408$. Magnification: 20x.
\end{abstract}

parental cell groups were 40/50, 49/50, and 49/50, respectively, at $24 \mathrm{~h}$ after transfection, and 5/50,40/50, and 49/50, respectively, at $39 \mathrm{~h}$ after transfection. Approximately 10/ 50 cells had lost GFP fluorescence in the Chol-miRCtrl group at $39 \mathrm{~h}$ after transfection. In addition, a few cells in which cytoplasmic Chol-let-7a was observed did not undergo cell death, and these cells subsequently lost their red fluorescence. This observation shows that some Chollet-7a-treated cells did not die.
Continuous observation of the images showed that cell proliferation and mobility decreased in the Chollet-7a- and Chol-miRCtrl-treated cells. In addition, images revealed that cell viability differed between the 2 control groups (Figure 2). There were no significant differences in proliferation and cell mobility between the 2 control groups immediately after Chol-miRCtrl transfection. However, the Chol-miRCtrl-treated cells exhibited poorer survival than the parental tumour cells

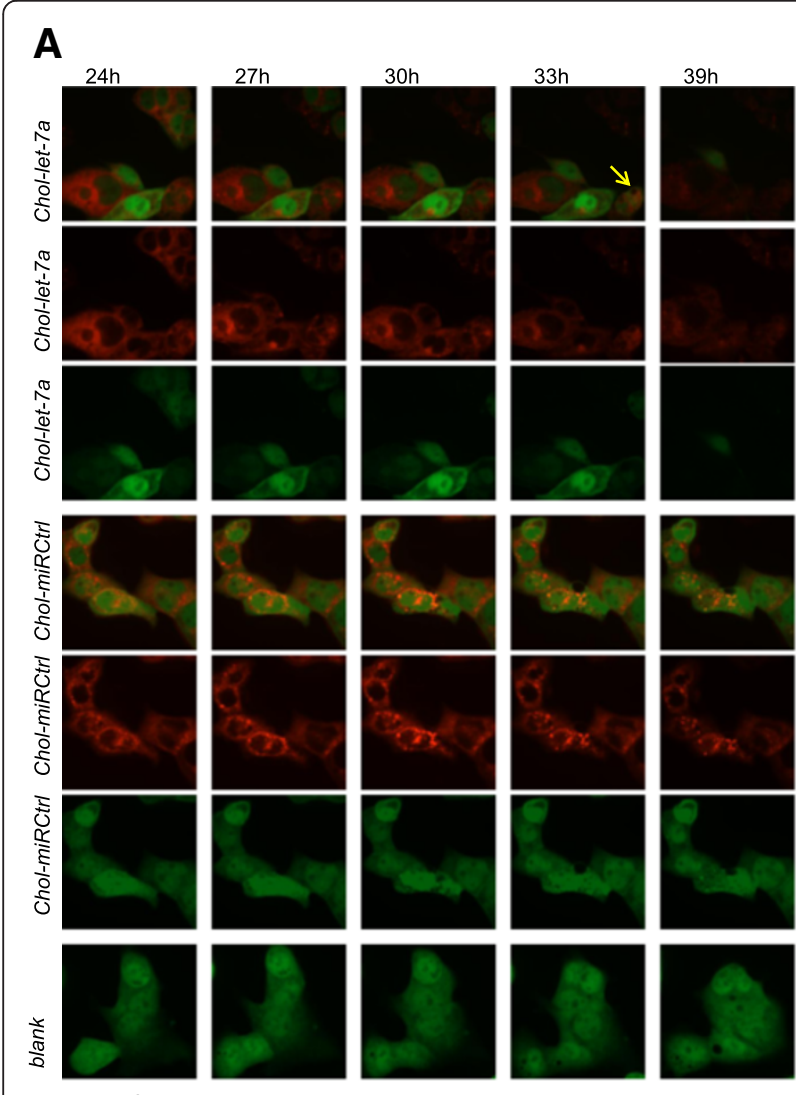

\section{B}

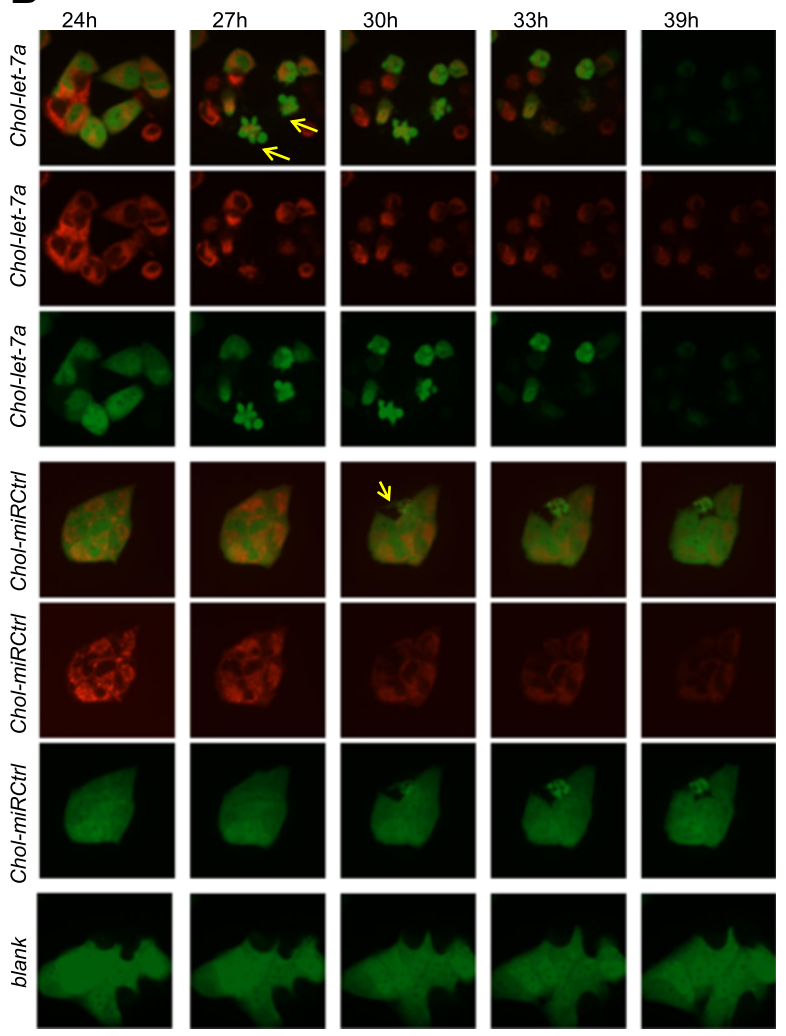

Magnification : $160 \times$

Figure 2 Live-cell images. Live-cell images were taken from $24 \mathrm{~h}$ to $39 \mathrm{~h}$ post-transfection. The parental cells with no treatment were observed as blank controls. Laser confocal images of GFP-labelled live cells (green) were recorded in the 3 groups. Cy5-labelled Chol-let-7a or Chol-miRCtrl appears as distinct red bodies. GFP-labelled living HepG2 or SMMC7721 cells appear green. Dead cells lose their green fluorescence. The images show more dead or apoptotic cells in Chol-let-7a-treated cells (yellow arrows). A: HepG2 cells. B: SMMC7721 cells. Magnification: 160×. 
(blank) from $39 \mathrm{~h}$ after transfection, and few living cells with GFP fluorescence were observed $72 \mathrm{~h}$ after treatment, whereas the parental cells were still active with respect to proliferation, growth, and mobility at this time point.

\section{Ultrastructure features}

We observed Chol-let-7a- and Chol-miRCtrl-treated cells under transmission electronic microscopy (TEM) at $48 \mathrm{~h}$ and $60 \mathrm{~h}$ post-transfection. Abnormal organelles were observed in the cytoplasm of Chol-let-7a-treated cells (Figure 3). Increased autophagocytic activity in HepG2 and SMMC7721 cells was observed $48 \mathrm{~h}$ after Chol-let$7 a$ treatment, as revealed by the presence of abundant lysosomes and phagolysosomes exhibiting heterolysosomes such as phagophores, multivesicular bodies (MVBs), and multilamellar bodies (MLBs) in the cytoplasm (Figure 3), but only slight changes in nuclear morphology were observed. Enlarged irregular mitochondria with disorganized mitochondrial crests and dilated rough endoplasmic reticulum (RER), which are often accompanied by degranulation, were also clearly observed in the Chol-let-7a-treated cells. Furthermore, vacuolated organelles were found in individual cells. Some changes observed in the Chol-let-7a-treated cells were also found in Chol-miRCtrl-treated HCC cells (see Additional file 3A); however, these effects were relatively mild in the negative control cells.

Long-term treatment produced significant ultrastructure modifications. In the cytoplasm of Chol-let-7atreated cells, mitochondria, heterolysosomes, and RER were vacuolated and showed irregular and unclear contours and structures (see Additional file 3B), and apoptotic and necrotic cells were clearly observed $60 \mathrm{~h}$ after treatment. In the Chol-miRCtrl group, a few cells underwent death. However, interestingly, cellular morphology did not show characteristics associated with apoptotic cells. Apoptotic nuclear changes, such as nuclear shrinkage and nuclear fragmentation, were barely observed

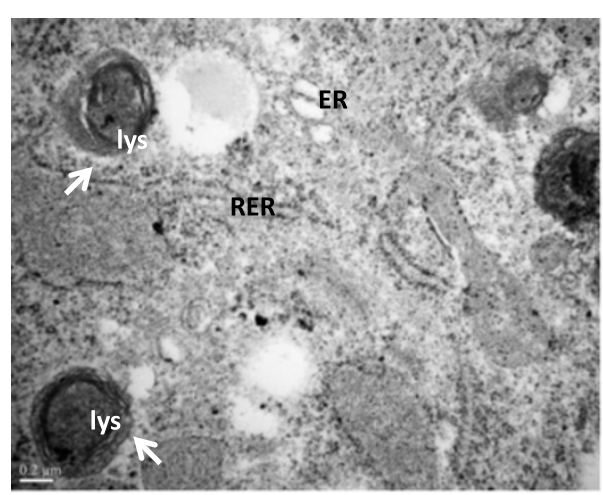

HepG2, Bar: $0.2 \mu \mathrm{m}$

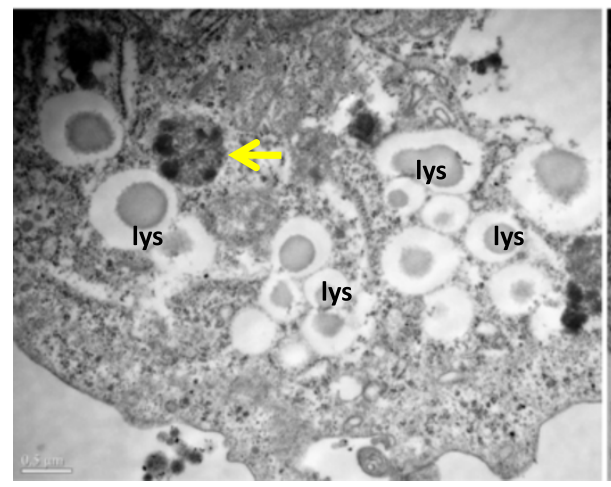

SMMC7721, Bar: $0.5 \mu \mathrm{m}$

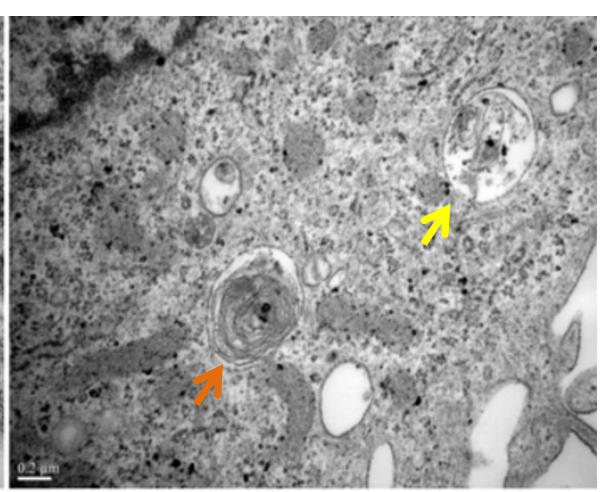

HepG2, Bar: $0.2 \mu \mathrm{m}$

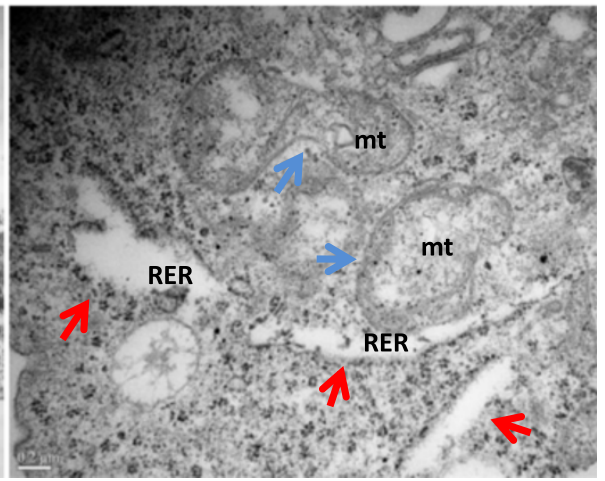

SMMC7721, Bar: $0.2 \mu \mathrm{m}$

Figure 3 Organelle changes after Chol-let-7a therapy under transmission electron microscopy. HepG2 and SMMC7721 cells were transfected with Chol-let-7a or the negative control miRNA mimic (Chol-miRCtr). Cells were collected at $48 \mathrm{~h}$ and $60 \mathrm{~h}$ and observed under TEM. The figure shows the cytoplasmic ultrastructure of Chol-let-7a-treated cells at $48 \mathrm{~h}$ post-transfection. Also shown are heterolysosomes as phagophores (white arrows), multivesicular bodies (MVBs, yellow arrows), and multilamellar bodies (MLBs, orange arrows). Mitophagosomes (double-membrane-enclosed damaged mitochondria) and enlarged irregular mitochondria ( $\mathrm{mt}$ ) are shown with blue arrows. Red arrows indicate the dilated RER with degranulation. Scale bars are $0.5 \mu \mathrm{m}$ or $0.2 \mu \mathrm{m}$. 
in Chol-let-7a-treated cells. In comparison with the rapid changes in cytoplasmic organelles, nuclear damage was strikingly delayed after Chol-let-7a-treatment (see Additional file 3C).

\section{Up-regulated let-7a down-regulated ras/RAS expression after systemic delivery}

To confirm that Chol-let-7a effectively carried let-7a to target tumours in vivo, we measured let-7a abundance in HepG2 orthotopic xenografts by qRT-PCR after systemic therapy. Using miRNA-specific primers, we found significant increases in let-7a miRNA abundance in treated xenografts (Figure 4A) (Chol-let-7a vs. Chol-miRCtrl, $p=0.008$; Chol-let-7a vs. blank, $p=0.013$; Chol-miRCtrl vs. blank, $p=0.128$ ). These results verified that Chol-let-7a successfully reached tumour tissues.

We analysed the expression of RAS proteins by western blotting and observed marked decreases in KRAS, HRAS, and NRAS abundance in Chol-let-7a-treated
A

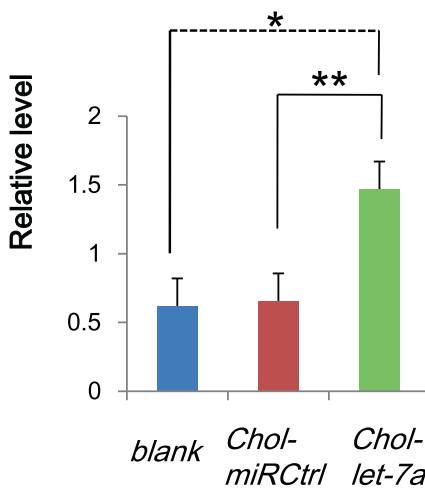

${ }^{*}: p<0.05 ;{ }^{* *} p<0.01$
B

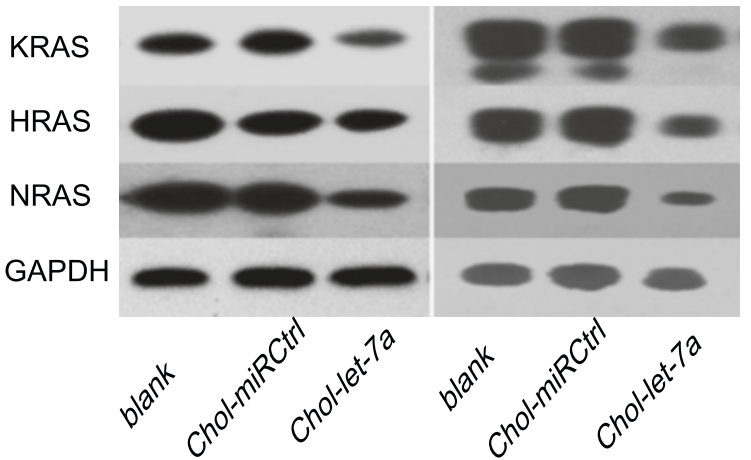

C
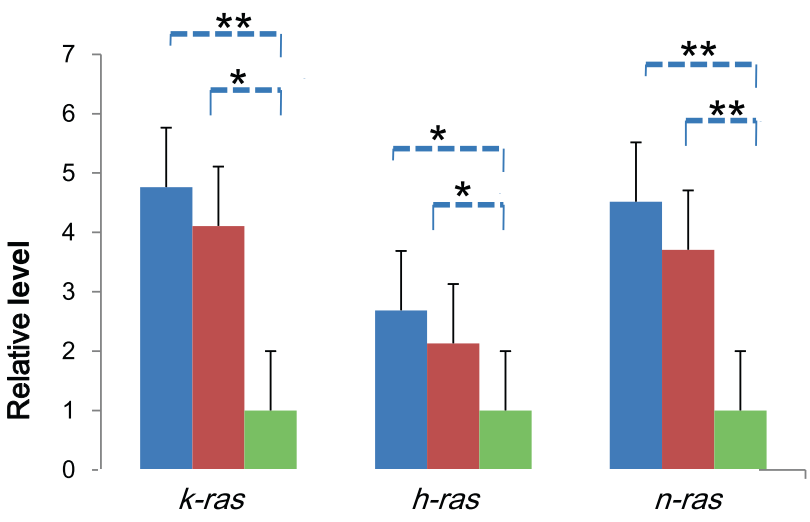

- blank

- Chol-miRCtrl

- chol-let-7a

*: $p<0.05 ;{ }^{* *} p<0.01$

Figure 4 Systemic Chol-let-7a therapy modulated ras/RAS abundance in HCC orthotopic xenografts. A: Let-7a expression in HepG2 orthotopic xenografts was examined by quantitative real-time PCR. Relative quantification of let-7a was calculated using the comparative cycle threshold $(C T)$ method $\left(2^{\Delta \Delta c t}\right)$ with let-7a normalized to U6. The results shown represent the mean and standard error from 3 independent experiments, ${ }^{*} p<0.05,{ }^{* *} p<0.01$ in comparison with controls. T-test: Chol-let-7a vs. Chol-miRCtrl, $p=0.008 ;$ Chol-let-7a vs. blank, $p=0.013 ;$ Chol-miRCtrl vs. blank, $p=0.128$. B: RAS protein expression in xenografts examined by western blotting. KRAS, HRAS, and NRAS protein expression was measured in xenografts by western blotting. Representative data are shown from 2 experiments. C: qRT-PCR analysis of ras mRNA in xenografts. Relative quantification of target genes was calculated using the comparative cycle threshold $(C T)$ method $\left(2^{\Delta \Delta c t}\right)$ with genes normalized to GAPDH. The results shown represent the mean and standard error from 3 independent experiments. ${ }^{*} p<0.05,{ }^{* *} p<0.01$ in comparison with controls. Analysis revealed deregulated expression of $k$-ras, $h$-ras, and $n$-ras mRNA. T-test: $n$-ras (Chol-let-7a vs. Chol-miRCtrl, $p=0.002$; Chol-let-7a vs. blank, $p=0.002$; Chol-miRCtrl vs. blank, $p=0.837$ ); $h$-ras (Chol-let-7a vs. Chol-miRCtrl, $p=0.016$; Chol-let-7a vs. blank, $p=0.033 ;$ Chol-miRCtrl vs. blank, $p=0.801$ ); and k-ras (Chol-let-7a vs. Chol-miRCtrl, $p=0.041$; Chol-let-7a vs. blank, $p=0.005$; Chol-miRCtrl vs. blank, $p=0.84$ ). 
xenografts (Figure 4B). Similarly, deregulated mRNA expression of $k$-ras, $h$-ras, and $n$-ras was also investigated by qRT-PCR. The expression of $n$-ras was inhibited most significantly by Chol-let-7a $(p<0.01)$, and expression levels of $h$-ras and $k$-ras $(p<0.05)$ were also reduced (Figure $4 C)$. These results suggest that Chol-let-7a successfully carried let-7a mimics into target HCC tumour cells and suppressed all 3 human ras genes at the transcriptional and translational levels, which were in accordance with our in vitro results.

\section{Chol-let-7a inhibited growth and metastasis of HCC orthotopic xenografts after systemic delivery}

To study antitumor efficacy in vivo, we examined the size of HepG2 orthotopic xenografts of different groups by ultrasound weekly after cell transplantation. As shown in Figure 5, the growth of orthotopic tumours was significantly inhibited following Chol-let-7a therapy (Figure 5A-C). One week after HepG2 cell transplantation, the xenografts (Volume, $\mathrm{mm}^{3}$ ) of the Chol-let-7a group $(8.2854 \pm 2.122593)$ were slightly larger than those of the 2 control groups (Chol-miRCtrl, $7.8614 \pm 1.69912$; blank, $7.0574 \pm 1.340323$ ), while the xenografts of the Chol-let-7a-treated group (152.1528 \pm 38.43266$)$ were significantly smaller than those of the 2 control groups (Chol-miRCtrl, 424.3472 $\pm 60.10395 ;$ blank, 380.2284 \pm 74.83116) at the culmination of therapy. The inhibitory rates produced by Chol-let-7a and Chol-miRCtrl on xenografts were $45.49 \%(p<0.01)$ and $-7.13 \%(p>0.05)$, respectively, in comparison with the blank control group that was treated with saline buffer alone.

Beginning 2 weeks after Chol-let-7a treatment, inhibition of tumour metastasis was observed by ultrasonography. Metastases within the liver are shown in Figure 5B. Local invasion and metastasis to the spleen were inhibited in the Chol-let-7a-treated group (data not shown).

Under light microscopy, small and large necrosis foci were observed in tumour tissues from all 3 groups, but more necrosis was observed in the Chol-let-7a-treated xenografts, and necrosis was also observed in capillaryrich areas. In contrast, significant necrosis was typically observed in the central tumour area in the control groups. These necrotic features were confirmed under TEM (Figure 5D). In addition, Chol-let-7a-treated tumour cells showed no significant atypia, and mitoses were very rare per unit of measurement in most areas in comparison with the control groups (Figure 5E). Immunohistochemical staining for Ki-67 and ultrastructure changes in HCC cells in the orthotopic xenografts showed similar features (data not shown). These results suggest that Chol-let-7a inhibits tumour growth by promoting cell death and inhibiting cell proliferation.

\section{Discussion}

We confirmed the significant antitumor efficacy of Chollet-7a on HCC, and in particular its significant effect on HepG2 orthotopic xenografts after systemic delivery in a preclinical animal model. Chol-let-7a effectively carried let-7a mimics to target tumours in vivo and inhibited tumour growth, metastasis within the liver, and local invasion and metastasis to the spleen. Significant increases in let-7a miRNA abundance were observed in Chol-let$7 a$-treated xenografts. Moreover, let-7a abundance in HepG2 orthotopic xenografts was coincident with a reduction in the expression of 3 human ras mRNAs and RAS proteins. These results suggest that Chol-let- $7 a$ inhibits HCC cell growth by regulating all 3 human ras genes at the transcriptional and translational levels.

We found some different features in the orthotopic xenograft tissues after Chol-let-7a systemic therapy in comparison with the 2 control groups. All tumour tissues contained small and large necrosis foci, but more necrosis was observed in the Chol-let-7a-treated xenografts, and necrosis was also observed in capillary-rich areas. In contrast, significant necrosis was typically observed in the central areas of tumours in the control groups. The histopathological features of Chol-let-7atreated xenografts may have been induced by the type of Chol-let-7a transportation used in this study. Tumour cells in capillary-rich areas could be more susceptible than other cells to systemically administered Chol-let-7a molecules. In addition, well-differentiated tumour cells with no significant atypia and only very rare mitoses were observed after Chol-let-7a therapy. These results suggest that Chol-let-7a inhibited tumour growth by inhibiting cell proliferation and promoting cell death.

We confirmed that Chol-let-7a entered cells and functioned primarily in the cytoplasm based on morphology and ultrastructure analysis. This result was consistent with the potential functional basis of let-7a, which involves the inhibition of target ras genes at the transcriptional and translational levels. In vitro, we observed the effects of Chol-let-7a on HCC cells by living cell image analysis and transmission electron microscopy. Both results suggested that Chol-let-7a entered cells and functioned primarily in the cytoplasm. The red fluorescence that indicated Chol-let-7a and Chol-miRCtrl was primarily focused in the cytoplasm. TEM revealed that Chol-let-7a damaged some cytoplasmic organelles, but only slight changes in nuclear morphology were observed. Cellular nuclear morphology did not show characteristics associated with apoptotic cells even at $60 \mathrm{~h}$ after Chollet-7a therapy, when long-term treatment had produced significant ultrastructure modifications in the cytoplasm. Apoptotic nuclear changes such as shrinkage and fragmentation were barely observed in Chol-let-7atreated cells, including those in which mitochondria, 

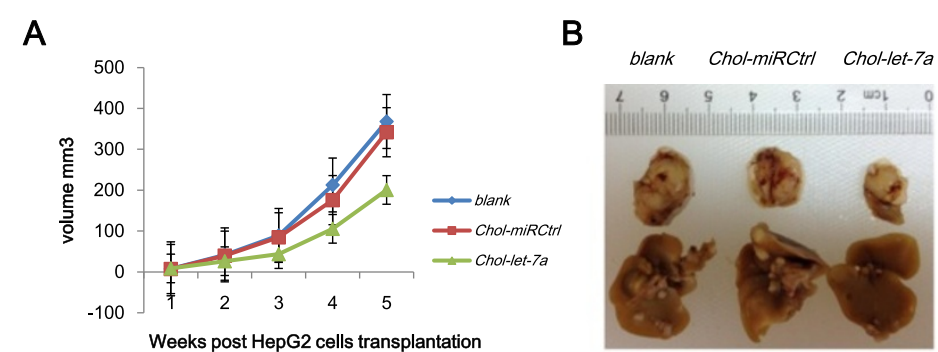

\section{C}

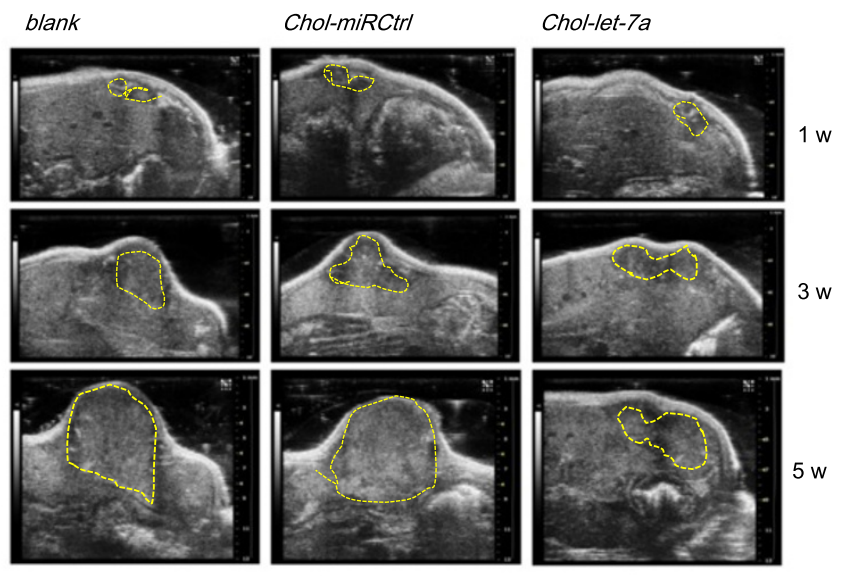

D blank

Chol-miRCtrl Chol-let-7a

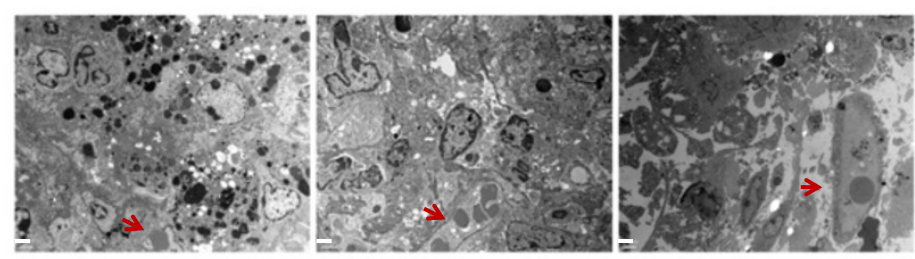

Bar: $2 \mu \mathrm{m}$

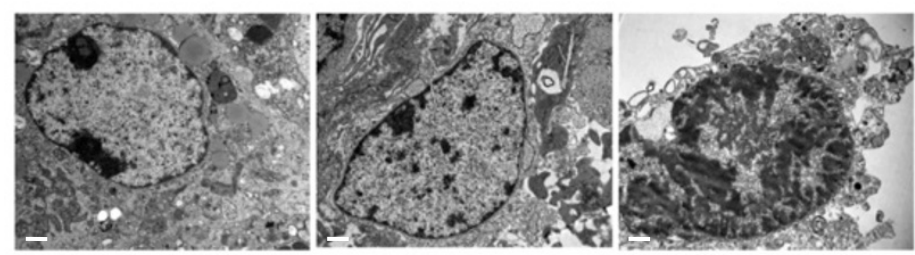

Bar: $0.5 \mu \mathrm{m}$

E

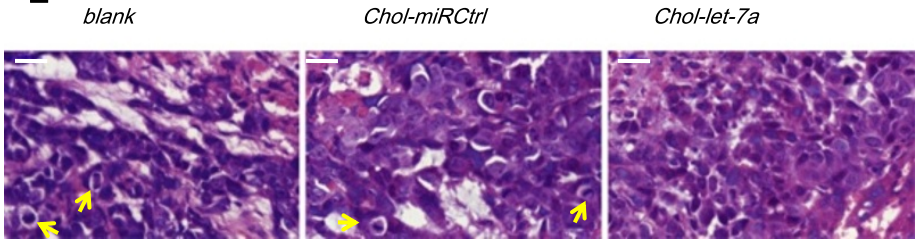

Bar: $20 \mu \mathrm{m}$

Figure 5 (See legend on next page.) 
(See figure on previous page.)

Figure 5 Systemic Chol-let-7a therapy inhibited growth and metastasis of orthotopic HepG2 xenografts. A: Xenograft growth curve. Representative xenografts from all groups are illustrated ( $N=6$ mice per cohort). The Chol-let-7a (green), Chol-miRCtrl (red), and blank control (blue) groups are shown. B: Macroscopic view of the HCC orthotopic tumour and metastasis within the liver of the treatment groups. C: Orthotopic HepG2 xenografts examined by ultrasonography. Xenograft images were recorded 1, 3, and 5 weeks after HepG2 cells were transplanted. D: TEM of HCC orthotopic tumours in vivo. Necrotic and tumour cells in HCC tissues are shown. Short red arrows indicate the microvasculature in orthotopic tumours. Scale bars are $2 \mu \mathrm{m}$ or $0.5 \mu \mathrm{m}$. E: H\&E staining of orthotopic HCC tumour tissues. H\&E staining was performed at the culmination of Chol-let-7a therapy. Differentiation and mitoses (short yellow arrows) of HCC cells of the 3 treatment groups are shown; all were recorded beside necrotic areas. Scale bars are $20 \mu \mathrm{m}$.

heterolysosomes, and RER were vacuolated and showed irregular and unclear contours and structures. In comparison with the rapid changes in cytoplasmic organelles, nuclear damage was strikingly delayed after Chol-let-7atreatment. Autophagocytic activity was observed in Chollet-7a- treated cells. Therefore, we suggest that autophagy may be an important mechanism through which Chol-let$7 a$ produces antitumor effects [25].

We previously examined the antitumor effect of Chollet-7a on HCC by using intratumoural administration in a subcutaneous xenograft model. Results showed that intratumoural administration of Chol-let-7a reduced tumour growth; however, cell phenotype and morphology in most areas of the subcutaneous xenografts showed no such changes, and these areas showed actively growing cells with high rates of mitosis (data not shown). Therefore, Chol-let-7a produces better inhibitory effects when it is systemically administered. Because of the high affinity of Chol-let-7a for liver tissue in nude mice (data not shown) and the convenience of systemic administration, Chol-let-7a represents a potential useful modified molecule for systemic HCC therapy.

However, the delivery system may have off-target effects, as indicated by the differences observed between the Chol-miRCtrl cells and the parental cells. Cell viability differed between the 2 control groups. The CholmiRCtrl-treated cells exhibited poorer survival than the parental tumour cells from $39 \mathrm{~h}$ after transfection, and few living cells with GFP fluorescence were observed $72 \mathrm{~h}$ after treatment, whereas the parental cells were still active with respect to proliferation, growth, and mobility at this time point. This result suggests that Chol-miRCtrl can reduce the viability of some cells. We compared the effects of Chol-let-7a and Chol-miRCtrl on HepG2 cells at 3 different doses. In comparison with the $25 \mathrm{nM}$ and $50 \mathrm{nM}$ doses, $100 \mathrm{nM}$ Chol-miRCtrl slightly slowed cell growth $72 \mathrm{~h}$ after transfection (see Additional file 4A), but there were no differences in cell growth between the treatment groups at $48 \mathrm{~h}$ (see Additional file 4B). These results suggest that dosage and prolonged action time contribute to the off-target effects of Chol-miRCtrl.

Ultrastructure features also differed between the 2 control groups. Some organelle changes observed in the
Chol-let-7a-treated cells were also found in the CholmiRCtrl-treated HCC cells under TEM. In addition, autophagy was observed in some Chol-miRCtrl treated tumour cells that under death, indicating that autophagocytic activity could also be a potential factor induces off-target effects. Given the observed high affinity of Chol-let-7a for liver tissue in nude mice, we hypothesize that Chol-let-7a may has potential off-target effects primary in liver tissue when it is administered systemically as a therapeutic molecule. In future studies, we will investigate off-target effects of Chol-let-7a in preclinical animal models.

\section{Conclusions}

We confirmed the significant antitumor efficacy of Chollet-7a on HCC, and in particular its significant effect on HepG2 orthotopic xenografts after systemic delivery in a preclinical animal model. Chol-let-7a effectively carried let-7a to target tumours in vivo and inhibit tumour growth by inhibiting cell proliferation and promoting cell death. In addition, Chol-let-7a can inhibit HCC cell growth by regulating all 3 human ras genes at the transcriptional and translational levels. Moreover, we confirmed Chol-let$7 a$ entered cells and functioned primarily in the cytoplasm, and autophagy may be an important mechanism through which Chol-let-7a produces antitumor effects. Taken together, Chol-let-7a represents a potential useful modified molecule for systemic HCC therapy. However, further studies of Chol-let-7a-produced off-target effects when it is systemically administered are required.

\section{Additional files}

Additional file 1: Up-regulated let-7a down-regulated human ras/
RAS expression in HCC cells in vitro. A: Let-7a levels measured by
quantitative real-time PCR $48 \mathrm{~h}$ post-transfection of Chol-let-7a or Chol-miRCtrl.
Significant increases in let-7a levels in Chol-let-7a-treated HepG2 and
SMMC7721 cells are shown. T-test: HepG2: Chol-let-7a vs. Chol-miRCtrl,
$p=0.003 ;$ Chol-let-7a vs. blank, $p=0.003 ;$ Chol-miRCtrl vs. blank, $p=0.08$.
SMMC7721: Chol-let-7a vs. Chol-miRCtrl, $p=0.001$; Chol-let-7a vs. blank,
$p=0.001$; Chol-miRCtrl vs. blank, $p=0.062$. The results shown represent
the mean and standard error from 3 independent experiments. $p<0.05$,
$* * 0.01$ in comparison with controls. (B) Expression of RAS proteins
examined by western blotting $48 \mathrm{~h}$ after transfection of Chol-let-7a or
Chol-miRCtrl. There was a marked decrease in KRAS, HRAS, and NRAS
protein abundance in Chol-let-7a-treated cells. (C) Deregulated expression


of $k$-ras, $h$-ras, and $n$-ras mRNAs as determined by qRT-PCR $48 \mathrm{~h}$ afte transfection of Chol-let-7a. T-test for k-ras in HepG2: Chol-let-7a vs. Chol-miRCtrl, $p=0.005$; Chol-let-7a vs. blank, $p=0.002$; Chol-miRCtrl vs, blank, $p=0.286$. T-test for k-ras in SMMC7721: Chol-let-7a vs. Chol-miRCtrl, $p=0.008 ;$ Chol-let-7a vs. blank, $p=0.007$; Chol-miRCtrl vs. blank, $p=0.463$ T-test for h-ras in HepG2: Chol-let-7a vs. Chol-miRCtrl, $p=0.005$; Chol-let-7a vs. blank, $p=0.001$; Chol-miRCtrl vs. blank, $p=0.081$. T-test for $h$-ras in SMMC7721: Chol-let-7a vs. Chol-miRCtrl, $p=0.032$; Chol-let-7a vs. blank, $p=0.023$; Chol-miRCtrl vs. blank, $p=0.907$. T-test for $n$-ras in HepG2: Chol-let-7a vs. Chol-miRCtrl, $p=0.001$; Chol-let-7a vs. blank, $p=0.004$ Chol-miRCtrl vs. blank, $p=0.755$. T-test for $n$-ras in SMMC7721: Chol-let-7a vs. Chol-miRCtrl, $p=0.001$; Chol-let-7a vs. blank, $p=0.002$; Chol-miRCtrl vs. blank, $p=0.958$. The results shown represent the mean and standard error from 3 independent experiments. ${ }^{*} p<0.05,{ }^{* *} p<0.01$ in comparison with controls.

Additional file 2: Live cell images showing apoptosis and distribution of Chol-let-7a and the negative control miRNA in HCC cells. The HCC cells were labelled with GFP. The cholesterol-conjugated let-7a mimics (Chol-let-7a), or negative control miRNA (Chol-miRCtr) were labelled with Cy5 fluorescence. Laser confocal images of GFP-labelled HepG2 and SMMC7721 cells (green) treated with 50 nM Cy5-labelled Chol-let-7a or Chol-miRCtrl are shown. The images are of HepG2 cells at 1 day after injection. Cy5 fluorescence appears in the cytoplasm as distinct red bodies surrounding the nucleus. Yellow arrow indicates an apoptotic cell in Chol-let-7a-treated group.

Additional file 3: HCC cells after Chol-let-7a or Chol-miRCtrl treatment observed by TEM. blank: Parental HCC cells; Chol-let-7a: Chol-let-7a-treated HCC cells; Chol-miRCtrl: Chol-miRCtrl-treated HCC cells A: HepG2 and SMMC7721 cells from the treatment groups at $48 \mathrm{~h}$ post-transfection. B: Chol-let-7a-treated cells at $60 \mathrm{~h}$ post-transfection. Vacuolated organelles with irregular and unclear contours and structures are shown.C: HCC cells observed under TEM. Parental, Chol-let-7a- and Chol-miRCtrl-treated HCC cells were observed under TEM at $48 \mathrm{~h}$ and $60 \mathrm{~h}$ after treatment. More dead and apoptotic cells were found in the Chol-let-7a-treated cells, but some Chol-miRCtrl-treated cells showed similar morphology.

\section{Additional file 4: MTT assay of HepG2 cells transfected with} different doses of Chol-let-7a or Chol-miRCtrl. HepG2 cells were transfected with $25 \mathrm{nM}, 50 \mathrm{nM}$, or $100 \mathrm{nM}$ of each treatment and the absorbance at $550 \mathrm{nM}$ was determined for each well at $48 \mathrm{~h}$ and $72 \mathrm{~h}$ after transfection. A: MTT assay of HepG2 cells at $72 \mathrm{~h}$ post-transfection with different doses of each treatment. At $72 \mathrm{~h}$, inhibition increased in a dose-dependent manner in the Chol-let-7a-treated group. In addition, cells transfected with $100 \mathrm{nM}$ Chol-miRCtrl (short arrow) grew more slowly than cells transfected with $25 \mathrm{nM}$ and $50 \mathrm{nM}$ Chol-miRCtrl. B: MTT assay of HepG 2 cells at $48 \mathrm{~h}$ post-transfection with different doses of each treatment. At $48 \mathrm{~h}$, HepG2 cells in the Chol-let-7a group were inhibited. In the Chol-let-7a-treated group, inhibition increased as the administered dose increased. No difference was observed between the Chol-miRCtrl-treated groups (long arrow) and the parental cells (blank).

\section{Competing interests}

The authors declare that they have no competing interests.

\section{Authors' contributions}

$J G$ initiated the project, ML and JG performed most of the experiments, YX performed ultrasonic analysis, WD and YH performed electronic microscopic analysis, JG and JC analysed data, and JG and ML wrote the manuscript. JC, $X Z, Y L, T Y, Z L$, and JC performed molecular imaging, western blotting, immunohistochemical staining, H\&E staining, and analysis. All authors read and approved the final manuscript.

\section{Acknowledgements}

We thank Mrs. Huimin Zhao, Wenyu Hao, and Huanxian Cui from the Centre for Experimental Animal Research (CEAR), Institute of Basic Medical Sciences, CAMS/PUMC, as well as Xiao Yang (VisualSonics, Inc., Beijing, China) and Yi Gao (Berthold, Beijing, China) for the technical support. We also thank Dr. Wei-Min Tong for his constructive suggestions in support of this study. We thank Dr. Xingyi Hang and Ms. Yuxing You for their assistance with the statistical analysis. This work was supported partly by the Scientific Data Sharing Program funded by the Chinese Ministry of Science (2004DKA20240-2013, JG, JC).

\section{Author details}

'Department of Pathology, Peking Union Medical College (PUMC) Hospital, PUMC \& Chinese Academy of Medical Sciences (CAMS), Beijing, China. ${ }^{2}$ Department of Ultrasound, Peking Union Medical College (PUMC) Hospital, PUMC \& Chinese Academy of Medical Sciences (CAMS), Beijing, China. ${ }^{3}$ The Core Laboratories Center, Institute of Basic Medical Sciences, PUMC \& CAMS, Beijing, China. ${ }^{4}$ National Laboratory of Medical Biology, Institute of Basic Medical Sciences, PUMC \& CAMS, Beijing, China. ${ }^{5}$ Department of Nuclear Medicine, Peking Union Medical College (PUMC) Hospital, PUMC \& Chinese Academy of Medical Sciences (CAMS), Beijing, China. ${ }^{6}$ Department of Scientific Research, Peking Union Medical College (PUMC) Hospital, PUMC \& Chinese Academy of Medical Sciences (CAMS), Beijing, China.

Received: 20 May 2014 Accepted: 23 October 2014

Published: 28 November 2014

\section{References}

1. Ferlay J, Shin HR, Bray F, Forman D, Mathers C, Parkin DM: Estimates of worldwide burden of cancer in 2008: GLOBOCAN 2008. Int I Cancer 2010, 127(12):2893-2917.

2. Lo CM, Ngan H, Tso WK, Liu CL, Lam CM, Poon RT, Fan ST, Wong J: Randomized controlled trial of transarterial lipiodol chemoembolization for unresectable hepatocellular carcinoma. Hepatology 2002, 35(5):1164-1171.

3. Wong CM, Kai AK, Tsang FH, Ng IO: Regulation of hepatocarcinogenesis by microRNAs. Front Biosci 2013, 5:49-60

4. Segura MF, Greenwald HS, Hanniford D, Osman I, Hernando E: MicroRNA and cutaneous melanoma: from discovery to prognosis and therapy. Carcinogenesis 2012, 33(10):1823-1832.

5. David S, Meltzer SJ: MicroRNA involvement in esophageal carcinogenesis. Curr Opin Pharmacol 2011, 11(6):612-616.

6. Lai EC: MicroRNAs are complementary to $3^{\prime} U T R$ sequence motifs that mediate negative post-transcriptional regulation. Nat Genet 2002, 30(4):363-364

7. Callegari E, Elamin BK, Sabbioni S, Gramantieri L, Negrini M: Role of microRNAs in hepatocellular carcinoma: a clinical perspective. Onco Targets Ther 2013, 6:1167-1178.

8. Xia H, Ooi LL, Hui KM: MicroRNA-216a/217-induced epithelial-mesenchymal transition targets PTEN and SMAD7 to promote drug resistance and recurrence of liver cancer. Hepatology 2013, 58(2):629-641.

9. Borel F, Konstantinova P, Jansen PL: Diagnostic and therapeutic potential of miRNA signatures in patients with hepatocellular carcinoma. J Hepatol 2012, 56(6):1371-1383.

10. Xia H, Ooi LL, Hui KM: MiR-214 targets $\beta$-catenin pathway to suppress invasion, stem-like traits and recurrence of human hepatocellular carcinoma. PLoS One 2012, 7(9):e44206. doi:10.1371/journal.pone.0044206.

11. Giordano S, Columbano A: MicroRNAs: new tools for diagnosis, prognosis, and therapy in hepatocellular carcinoma? Hepatology 2013, 57(2):840-847.

12. Johnson SM, Grosshans H, Shingara J, Byrom M, Jarvis R, Cheng A, Labourier E, Reinert KL, Brown D, Slack FJ: RAS is regulated by the let-7 microRNA family. Cell 2005, 120(5):635-647.

13. Torrisani J, Bournet B, du Rieu MC, Bouisson M, Souque A, Escourrou J, Buscail L, Cordelier P: let-7 MicroRNA transfer in pancreatic cancerderived cells inhibits in vitro cell proliferation but fails to alter tumor progression. Hum Gene Ther 2009, 20(8):831-844.

14. He XY, Chen JX, Zhang Z, Li CL, Peng QL, Peng HM, He XY, Chen JX, Zhang Z, Li C, Peng QL, Peng HM: The let-7a microRNA protects from growth of lung carcinoma by suppression of $\mathrm{k}$-Ras and c-Myc in nude mice. J Cancer Res Clin Oncol 2010, 136(7):1023-1028.

15. Taketomi A, Shirabe K, Muto J, Yoshiya S, Motomura T, Mano Y, Ikegami T, Yoshizumi T, Sugio K, Maehara Y: A rare point mutation in the Ras oncogene in hepatocellular carcinoma. Surg Today 2013, 43(3):289-292. doi:10.1007/500595-012-0462-8.

16. Calvisi DF, Ladu S, Gorden A, Farina M, Conner EA, Lee JS, Factor VM, Thorgeirsson SS: Ubiquitous activation of Ras and Jak/Stat pathways in human HCC. Gastroenterology 2006, 130(4):1117-1128. 
17. Calvisi DF, Ladu S, Conner EA, Seo D, Hsieh JT, Factor VM, Thorgeirsson SS: Inactivation of Ras GTPase-activating proteins promotes unrestrained activity of wild-type Ras in human liver cancer. J Hepatol 2011, 54(2):311-319. doi:10.1016/j.jhep.2010.06.036.

18. Trang P, Wiggins JF, Daige CL, Cho C, Omotola M, Brown D, Weidhaas JB, Bader AG, Slack FJ: Systemic delivery of tumor suppressor microRNA mimics using a neutral lipid emulsion inhibits lung tumors in mice. Mol Ther 2011, 19(6):1116-1122. doi:10.1038/mt.2011.48.

19. Fischer S, Wagner A, Kos A, Aschrafi A, Handrick R, Hannemann J, Otte K Breaking limitations of complex culture media: Functional non-viral miRNA delivery into pharmaceutical production cell lines. J Biotechnol 2013, 168(4):589-600.

20. Zhang J, Sun Q, Zhang Z, Ge S, Han ZG, Chen WT: Loss of microRNA-143/145 disturbs cellular growth and apoptosis of human epithelial cancers by impairing the MDM2-p53 feedback loop. Oncogene 2013, 32(1):61-69.

21. Hou J, Lin L, Zhou W, Wang Z, Ding G, Dong Q, Qin L, Wu X, Zheng Y, Yang Y, Tian W, Zhang Q, Wang C, Zhang Q, Zhuang SM, Zheng L, Liang A, Tao W, Cao X: Identification of miRNomes in human liver and hepatocellular carcinoma reveals miR-199a/b-3p as therapeutic target for hepatocellular carcinoma. Cancer Cel 2011, 19(2):232-243. doi:10.1016/j.ccr.2011.01.001.

22. Ji J, Zhao L, Budhu A, Forgues M, Jia HL, Qin LX, Ye QH, Yu J, Shi X, Tang $Z Y$, Wang $X W$ : Let-7g targets collagen type I a2 and inhibits cell migration in hepatocellular carcinoma. J Hepatol 2010, 52(5):690-697.

23. Livak KJ, Schmittgen TD: Analysis of relative gene expression data using real-time quantitative PCR and the 2(-DeltaDeltaC(T)) Method. Methods 2001, 25(4):402-408.

24. Feldmann G, Fendrich V, McGovern K, Bedja D, Bisht S, Alvarez H, Koorstra JB, Habbe N, Karikari C, Mullendore M, Gabrielson KL, Sharma R, Matsui W, Maitra A: An orally bioavailable small-molecule inhibitor of Hedgehog signaling inhibits tumor initiation and metastasis in pancreatic cancer. Mol Cancer Ther 2008, 7:2725-2735.

25. Levine B, Klionsky DJ: Development by self-digestion: molecular mechanisms and biological functions of autophagy. Dev Cell 2004, 6:463-477.

doi:10.1186/1471-2407-14-889

Cite this article as: Liu et al.: Cholesterol-conjugated let-7a mimics: antitumor efficacy on hepatocellular carcinoma in vitro and in a preclinical orthotopic xenograft model of systemic therapy. BMC Cancer 2014 14:889

\section{Submit your next manuscript to BioMed Central and take full advantage of:}

- Convenient online submission

- Thorough peer review

- No space constraints or color figure charges

- Immediate publication on acceptance

- Inclusion in PubMed, CAS, Scopus and Google Scholar

- Research which is freely available for redistribution 